\title{
Salmonella - at home in the host cell
}

\section{Preeti Malik-Kale, Carrie E. Jolly, Stephanie Lathrop, Seth Winfree, Courtney Luterbach and Olivia Steele-Mortimer*}

Laboratory of Intracellular Parasites, Rocky Mountain Laboratories, National Institutes of Allergy and Infectious Disease, National Institute of Health, Hamilton, MT, USA

\author{
Edited by: \\ John S. Gunn, The Ohio State \\ University, USA \\ Reviewed by: \\ Gregory Plano, University of Miami \\ Miller School of Medicine, USA \\ Tim Yahr, University of lowa, USA \\ *Correspondence: \\ Olivia Steele-Mortimer, Rocky \\ Mountain Laboratories, National \\ Institutes of Allergy and Infectious \\ Disease, National Institute of Health, \\ 903 South 4th Street, Hamilton, MT \\ 59840, USA. \\ e-mail: omortimer@niaid.nih.gov
}

\begin{abstract}
The Gram-negative bacterium Salmonella enterica has developed an array of sophisticated tools to manipulate the host cell and establish an intracellular niche, for successful propagation as a facultative intracellular pathogen. While Salmonella exerts diverse effects on its host cell, only the cell biology of the classic "trigger"-mediated invasion process and the subsequent development of the Salmonella-containing vacuole have been investigated extensively. These processes are dependent on cohorts of effector proteins translocated into host cells by two type III secretion systems (T3SS), although T3SS-independent mechanisms of entry may be important for invasion of certain host cell types. Recent studies into the intracellular lifestyle of Salmonella have provided new insights into the mechanisms used by this pathogen to modulate its intracellular environment. Here we discuss current knowledge of Salmonella-host interactions including invasion and establishment of an intracellular niche within the host.
\end{abstract}

Keywords: effectors, invasion, membrane tubules, phagosome, type III secretion system, vacuole

\section{INTRODUCTION}

Salmonella enterica are facultative intracellular pathogens that are found in the gastrointestinal tract of mammalian, avian, and reptilian hosts. These Gram-negative bacteria are highly versatile and can adapt to a wide range of conditions both in the natural environment and within host organisms. While there are more than 2,500 S. enterica serovars only a few are commonly associated with disease in mammals. In humans, Salmonella are primarily associated with either localized intestinal infection or severe systemic disease. Salmonella gastroenteritis is usually self-resolving in healthy adults. It is one of the most common causes of foodborne disease, possibly affecting over 90 million people globally each year (Majowicz et al., 2010), and can be caused by many serovars although the most common are serovars Typhimurium and Enteritidis. Systemic disease in healthy humans (typhoid) is caused by serovar Typhi and a handful of other serovars that are strictly adapted to humans and higher primates. Immunocompromised individuals, such as those with AIDS or cancer, often develop systemic salmonellosis when infected with non-typhoidal Salmonella serovars (Gordon, 2008).

The interplay between Salmonella and its vertebrate hosts is complex and involves a variety of virulence factors, although two of the most important are the type III secretion systems 1 and 2 (T3SS1 and T3SS2). Together these are used to inject over 30 effector proteins into the cytoplasm of host cells where they act on a variety of pathways. In epithelial cells, T3SS effectors are essential for both invasion and the subsequent establishment of the intracellular niche by Salmonella (Figure 1). The intracellular niche is a modified phagosome, known as the Salmonella-containing vacuole (SCV), which undergoes extensive T3SS effector-dependent membrane remodeling. This review focuses on how Salmonella establish their intracellular niche in epithelial cells with particular emphasis on invasion and SCV biogenesis.

\section{SALMONELLA TYPE III SECRETION SYSTEMS}

Type III secretion systems are sophisticated contact-dependent delivery systems used by many Gram-negative bacterial pathogens to inject bacterial effector proteins into host cells. These nanoinjection systems consist of 20-30 proteins, many of which have homology to proteins in the flagellar export apparatus [for review (Marlovits and Stebbins, 2010)]. While all T3SSs are structurally similar, the effectors secreted by these delivery systems are extremely diverse (Samudrala et al., 2009). T3SS1 and T3SS2 are encoded on different regions of the chromosome, known as Salmonella pathogenicity islands 1 and 2 (SPI1 and SPI2) respectively, and are functionally and temporally distinct. The SPI1-encoded T3SS1 translocates a cohort of effectors that drive "trigger"mediated invasion of host cells whereas the SPI2-encoded T3SS2 is induced after invasion and is required for modulation of the intracellular environment. Nevertheless, it is now apparent that some overlap exists and effectors from both systems mediate biogenesis of the SCV (Hernandez et al., 2004; Drecktrah et al., 2005; Lawley et al., 2006; Brawn et al., 2007).

\section{SALMONELLA ENTRY}

In vivo, Salmonella can be found in a variety of phagocytic and non-phagocytic cells, including macrophages, dendritic cells, neutrophils, M cells, and enterocytes (Wallis et al., 1986; Jones et al., 1994; Richter-Dahlfors et al., 1997; Rescigno et al., 2001; Salcedo et al., 2001; Meyerholz et al., 2002; Geddes et al., 2007). Bacterial internalization, whether by phagocytosis or Salmonella-mediated invasion, involves actin remodeling at its core, which results in formation of plasma membrane extensions and ingestion of the target particle into the membrane bound phagosome. Common features of this process are the involvement of Rho family GTPases and phosphoinositides, which are instrumental in actin remodeling, membrane trafficking and signal transduction. The Rho 


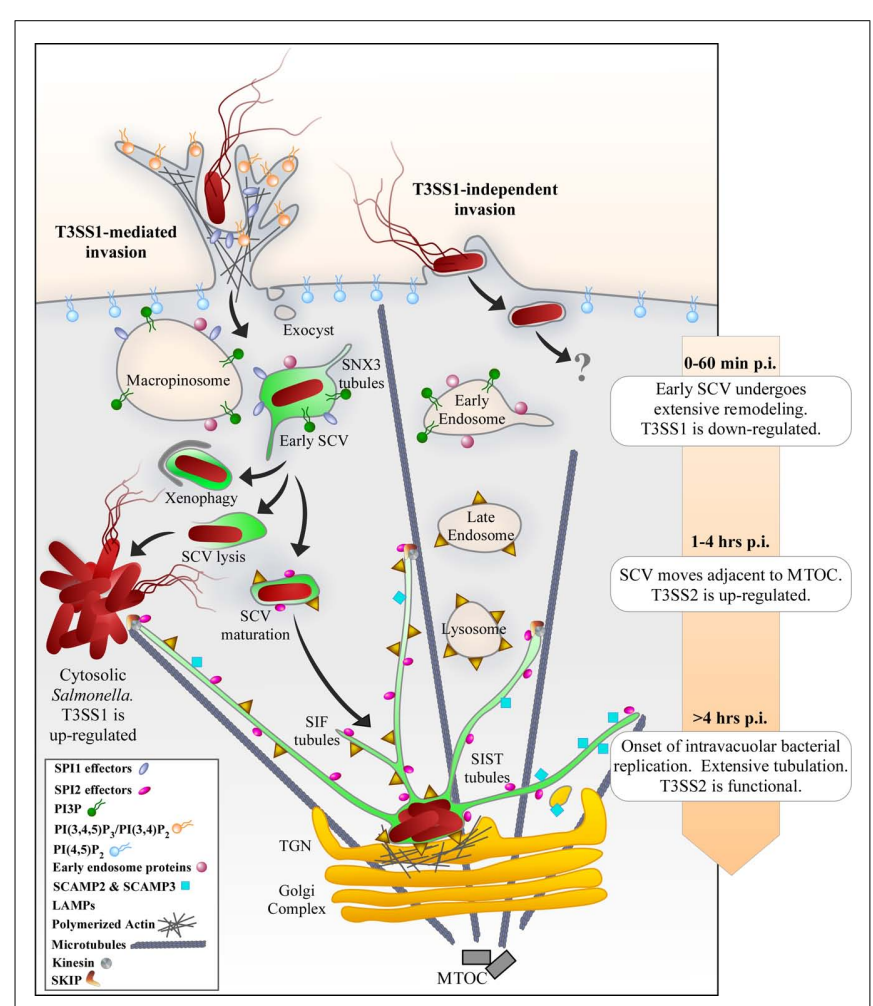

FIGURE 1 | Biogenesis of the SCV. Invasive Salmonella use T3SS1 to translocate effector proteins into host cells. Several of these effectors drive actin-mediated ruffling and internalization of the bacteria into a modified phagosome or SCV. T3SS1 effectors are also present on the SCV membrane and are important for rapid remodeling of the membranes as well as more sustained effects. The early SCV has many characteristics of early endosomes, including the phospholipid PI(3)P and proteins that interact with it such as Rab5 and SNX1. Dynamic tubular networks containing SNX1 or SNX3 are involved in membrane remodeling during this early stage in SCV biogenesis. During this initial phase of infection the majority of Salmonella down-regulate T3SS1 and induce T3SS2, which is required for subsequent steps in SCV biogenesis. The majority of SCVs relocate to a juxtanuclear location within $1-2 \mathrm{~h}$ and become enriched in proteins, such as Lamp1, Rab7, and vacuolar ATPase, that are normally found in late endosomes and lysosomes. However, some SCVs do not undergo this maturation process and instead either lyse and release the bacteria into the cytosol or are targeted by the autophagy system. In the mature SCV replication is initiated 4-6 h post invasion and is accompanied by the formation of a dynamic tubular network that extends from the surface of the SCV. Tubules enriched in Lamp1 are known as Sifs although another population of Lamp1-ve tubules (SISTs) has recently been described. In epithelial cells cytosolic Salmonella replicate to high numbers, compared to bacteria inside SCVs, and become re-induced for T3SS1 and flagella. Salmonella can also invade cells via T3SS1-independent mechanisms (right side), although biogenesis of the SCV under these conditions has not been well studied.

GTPases, Cdc42, and Rac1, are required for the initiation of actin polymerization during Fc gamma receptor-mediated phagocytosis as well as for Salmonella invasion (Zhou and Galan, 2001; Hoppe and Swanson, 2004; Scott et al., 2005). Remodeling of F-actin during phagocytosis is also dependent on phosphatidylinositol $(4,5)$ bisphosphate $\left[\mathrm{PI}(4,5) \mathrm{P}_{2}\right]$ a phospholipid with multiple roles in the nucleation, elongation, and bundling of actin filaments (Hilpela et al., 2004; Scott et al., 2005). Local changes in membrane phospholipid composition can also affect the net charge on the cytoplasmic surface of membranes resulting in the selective recruitment of proteins such as members of the Rho family of GTPases (Magalhaes and Glogauer, 2010).

Bacterial internalization is accompanied by changes in host cell signaling pathways, affecting a number of vital cellular processes, including membrane trafficking, cell division, apoptosis, microbial killing, cytokine production, and antigen presentation. The ultimate fate of intracellular Salmonella is determined by a complex interplay of both host and bacterial factors. Here we focus on the entry methods employed by Salmonella invasion of non-phagocytic epithelial cells.

\section{T3SS1-DEPENDENT INVASION}

Type III secretion systems 1-mediated invasion of non-phagocytic cells by Salmonella has been extensively studied. Morphologically, this "trigger"-mediated invasion process is characterized by the extremely rapid appearance of membrane ruffles on the surface of the host cell, and subsequent formation of spacious phagosomes/vacuoles, or macropinosomes, which may or may not contain bacteria. These events are mediated by a subset of T3SS1 effectors (SipA, SipC, SopB, SopE, SopE2) that act in concert to induce massive localized rearrangements of actin and the plasma membrane as well as activating signaling pathways [for review (McGhie et al., 2009; Dunn and Valdivia, 2010)]. While SipA and SipC directly bind actin (McGhie et al., 2001; Hayward and Koronakis, 2002), SopB, SopE, and SopE2 indirectly modulate actin activity by stimulating Rho family GTPases, which are common targets of bacterial T3SS effectors.

SipA stabilizes actin filaments by enhancing the localized actinbundling activity of T-plastin (Zhou et al., 1999) while also inhibiting the filament-severing activities of cofilin and gelsolin (McGhie et al., 2004). SipC can independently cause actin rearrangements via its distinct actin-bundling and actin-nucleating domains (Hayward and Koronakis, 1999; Myeni and Zhou, 2010). In addition to modulating actin, SipC interacts directly with Exo70, a component of the exocyst complex, which mediates docking and fusion of exocytic vesicles with the plasma membrane (Nichols and Casanova, 2010). SopB (also known as SigD) is a phosphoinositide phosphatase, which can hydrolyse a number of phosphoinositides and inositol phosphates in vitro and has diverse effects on host cells (Norris et al., 1998; Steele-Mortimer et al., 2000; Knodler et al., 2005; Dukes et al., 2006; Patel and Galan, 2006; Dai et al., 2007; Bujny et al., 2008; Mallo et al., 2008; Patel et al., 2009; Bakowski et al., 2010; Braun et al., 2010). During invasion, SopB participates in actin remodeling by activating RhoG (Patel and Galan, 2006). In its active state RhoG recruits the ELMO-Dock180 complex to the membrane to promote Rac activation and has been implicated in membrane ruffling and engulfment (Gauthier-Rouviere et al., 1998; Katoh and Negishi, 2003). SopB does not directly activate RhoG but instead targets a guanine-nucleotide exchange factor (GEF), known as SH3-containing GEF (SGEF) that promotes the exchange of GDP for GTP on RhoG (Patel and Galan, 2006). Although SopB phosphatase activity is required for SGEF activation the mechanism remains undefined (Raffatellu et al., 2005; Patel and Galan, 2006). 
Whereas SopB activates a GEF, SopE and SopE2 act by mimicking mammalian GEFs. These effectors are members of a family of bacterial GEFs, which bind to Rho GTPases including Racl and Cdc42, and activate them by catalyzing exchange of GDP for GTP (Hardt et al., 1998; Stevens et al., 2003). SopE and SopE2 are about 70\% identical (Stender et al., 2000), and have different specificities and distribution amongst S. enterica serovars. SopE2 appears to be specific for Cdc42 whereas SopE acts on Cdc42 and Rac1. Interestingly, the requirement for $\mathrm{Cdc} 42$, but not $\mathrm{Racl}$, is dispensable for Salmonella invasion in some model systems (Criss et al., 2001; Patel and Galan, 2006).

Recent work suggests that SopE can also activate RalA, a GTPase that is required for assembly of the exocyst (Nichols and Casanova, 2010) and has been implicated in Fc gamma receptormediated phagocytosis (Corrotte et al., 2010). Thus SopE and SipC together appear to direct fusion of exocytic vesicles with the plasma membrane at the site of entry, presumably as a source of membrane for the expanding ruffle or phagocytic cup (Nichols and Casanova, 2010). SopB may also stimulate membrane fusion at the site of entry by increasing local levels of $\mathrm{PI}(3) \mathrm{P}$ resulting in the plasma membrane recruitment of VAMP8, a member of the endosomal synaptobrevin/VAMP family that mediates homotypic fusion of early endosomes (EE; Pryor et al., 2004; Dai et al., 2007). Thus, Salmonella T3SS1-mediated invasion may involve fusion of several intracellular sources of membrane at the site of entry.

In the context of this review, it is worth mentioning several non-receptor kinases implicated in T3SS1-induced actin reorganization and/or downstream signaling. These include Abl, Ack, and the focal adhesin kinase FAK (Murli et al., 2001; Shi and Casanova, 2006; Ly and Casanova, 2009). The signal from these kinases is further mediated to the actin cytoskeleton by scaffold proteins such as p130Cas/BCAR1 (Shi and Casanova, 2006), Shank3 (Huett et al., 2009), and IQGAP1 (Brown et al., 2007), during Salmonella invasion. p130Cas/BCAR1 is a member of the Crk-associated substrate (Cas) scaffolding protein family that interacts with FAK (Petch et al., 1995; Schuetz et al., 2004) and is involved in membrane ruffling (Sharma and Mayer, 2008). Intriguingly, IQGAP1 also interacts with Exo70 and it has been implicated in exocyst localization and exocytosis (Rittmeyer et al., 2008).

\section{T3SS1-INDEPENDENT INVASION}

Type III secretion systems-independent mechanisms may be important for invasion of specific cell types or under certain conditions (Heffernan et al., 1992b; Aiastui et al., 2010; Radtke et al., 2010; Rosselin et al., 2010). In the absence of T3SS1 at least two non-fimbrial outer membrane proteins (Omps), Rck and PagN, can mediate zipper-like invasion of non-phagocytic cells. Both of these Omps, when expressed in non-invasive E. coli strains, confer the ability to bind to and invade fibroblastic, epithelial, and endothelial cells, although the efficiency of invasion is cell type dependent (Heffernan et al., 1992b; Lambert and Smith, 2008, 2009; Rosselin et al., 2010). Rck is a $19 \mathrm{kDa}$ member of a family of virulence-associated Omps expressed in Gram-negative bacteria, which promote internalization in epithelial cells and internalization and survival in macrophages (Heffernan et al., 1992a; Cirillo et al., 1996). Rck-mediated entry is dependent upon the Arp2/3 complex and Rac1 and Cdc42 which mobilize actin rearrangements resulting in membrane ruffling and bacterial uptake (Unsworth et al., 2004; Rosselin et al., 2010). PagN is a $26 \mathrm{kDa}$ protein with similarity to the known invasins/adhesins, Hek and Tia, of pathogenic E. coli (Lambert and Smith, 2008). The binding of PagN to cell surface heparin sulfate proteoglycans mediates both adhesion and invasion of Salmonella (Lambert and Smith, 2009).

Each Salmonella serovar has over a dozen adhesin gene clusters many of which are implicated in pathogenesis (Edwards et al., 2002; Clayton et al., 2008). Included in this group are distinct types of fimbriae (type I fimbriae, plasmid-encoded fimbriae, long polar fimbriae, thin aggregative fimbriae) and non-fimbrial adhesins (ShdA and SiiE). While type I fimbriae can mediate binding to epithelial cells, they also help induce actin-dependent uptake in the absence of T3SS1 (Misselwitz et al., 2010). SiiE is a giant adhesin that is required for efficient T3SS1-mediated invasion into polarized epithelial cells but not into non-polarized epithelial cells or fibroblasts (Gerlach et al., 2008).

Although T3SS1-mediated invasion has been the center of attention for over a decade the recent use of alternative in vitro model systems have made it evident that Salmonella can use multiple ways to invade non-phagocytic host cells. It is not clear why Salmonella has developed alternate mechanisms for cellular invasion, but it is hypothesized that these Omps and/or adhesins when used in conjunction with T3SS1-mediated invasion may help provide cell and host specificity. While none of these mechanisms rival T3SS1-mediated invasion in complexity and efficiency they may certainly play an important roles in Salmonella pathogenesis.

\section{LIFE IN A VACUOLE}

Following internalization into the host cell Salmonella are located in the SCV, a vacuolar niche modified and adapted by the bacteria via the activities of both T3SS1 and T3SS2 effectors (Figure 1). The newly formed SCV is enriched in EE proteins, such as EEA1, which are subsequently replaced by late endosomal/lysosomal (LE/lys) markers, such as the lysosomal glycoprotein Lamp1 (Garcia-del Portillo and Finlay, 1995; Steele-Mortimer et al., 1999). In cultured epithelial cells, the SCV migrates from the cell periphery to a juxtanuclear position within $1-2 \mathrm{~h}$ following invasion (Abrahams et al., 2006; Ramsden et al., 2007). Bacterial replication is initiated approximately $4-6 \mathrm{~h}$ post invasion and is accompanied by the extension of Lamp1 enriched membrane tubules (Sifs) from the surface of the SCV (Garcia-del Portillo et al., 1993). T3SS1 effectors play an important role, not only in invasion but also in early stages of SCV biogenesis. In contrast T3SS2, which is induced intracellularly following invasion, is required for later events in SCV biogenesis including the onset of bacterial replication and Sif formation (Waterman and Holden, 2003). Recent studies, regarding the roles of T3SS effectors and host proteins in SCV biogenesis, have provided new insights into the mechanisms used by Salmonella to establish its intracellular niche. Here we highlight some of these findings and discuss their significance. For convenience, SCV biogenesis is separated into three stages: early, intermediate, and late. 


\section{EARLY VACUOLE DEVELOPMENT - T3SS1 EFFECTORS, SPACIOUS VACUOLES, AND TUBULES}

Immediately after formation, the SCV undergoes rapid membrane remodeling (Figure 1) predominantly driven by the activities of the T3SS1 effector SopB, which is translocated during entry and then persists in the host cell for several hours following invasion (Kubori and Galan, 2003; Drecktrah et al., 2005). During initial contact with the host plasma membrane, the phosphoinositide phosphatase activity of SopB manipulates plasma membrane phosphoinositides to activate Akt via $\mathrm{PI}(3,4) \mathrm{P}_{2}$ and/or PI(3,4,5) $\mathrm{P}_{3}$ (Steele-Mortimer et al., 2000) and also drives macropinosomes/phagosome formation via removal of $\mathrm{PI}(4,5) \mathrm{P}_{2}$ (Terebiznik et al., 2002). Thereafter, SopB located on the cytosolic face of the SCV directs an increase in PI(3)P, a phosphoinositide normally associated with $\mathrm{EE}$, and a decrease in $\mathrm{PI}(4,5) \mathrm{P}_{2}$ (Hernandez et al., 2004; Mallo et al., 2008; Bakowski et al., 2010). SopB-dependent acquisition of $\mathrm{PI}(3) \mathrm{P}$ is not directly driven by SopB phosphatase activity but rather a result of SopB-dependent recruitment of the small GTPase Rab5 to the SCV membrane (Hernandez et al., 2004; Mallo et al., 2008). As a consequence the Rab5 interacting protein Vps34 is also recruited, and it is the activity of this type III PI3K that generates PI(3)P in the SCV membrane (Mallo et al., 2008). SopB activity is also required for reducing levels of negatively charged $\mathrm{PI}(4,5) \mathrm{P}_{2}$ and phosphatidylserine from the SCV, which results in the dissociation of several Rab proteins from the SCV and may serve to delay SCV-lysosome fusion (Hernandez et al., 2004; Mallo et al., 2008; Bakowski et al., 2010).

Phosphoinositide binding proteins, such as the sorting nexins (SNX), are important regulators of membrane trafficking. The SNX protein family comprises over 30 members, each of which contains a Phox homology (SNX-PX) domain that binds to membrane phosphoinositides (Worby and Dixon, 2002). Two SNX proteins, SNX1 and SNX3, participate in early SCV biogenesis in a SopB-dependent manner (Bujny et al., 2008; Braun et al., 2010). Many SNX proteins, including SNX1, also contain a BAR (Bin-amphiphysin-Rvs) domain, which can sense or induce membrane curvature (Van Weering et al., 2010). In SNX1 the PX and BAR domains, co-operatively target the protein to specific parts of the EE, enriched in $\mathrm{PI}(3) \mathrm{P}$ and $\mathrm{PI}(3,5) \mathrm{P}_{2}$, where they promote tubule formation (Carlton et al., 2004). SNX1 is a member of the retromer sorting complex that mediates retrieval of receptors from the endolysosomal pathway to the TGN, a process that is believed to be mediated by membrane tubules (Carlton et al., 2004). During Salmonella invasion of epithelial cells SNX1 first accumulates in the proximity of membrane ruffles and then, within 15 min post invasion, localizes to tubules that emanate from the SCV and are associated with vacuolar shrinking (Bujny et al., 2008). In SNX1 depleted cells, movement of the SCV to the juxtanuclear region and onset of replication are delayed and the cation-independent mannose-6-phosphate receptor (CI M6PR) accumulates on SCVs (Bujny et al., 2008). CI M6PR is used for delivery of soluble lysosomal enzymes to lysosomes and is normally excluded from SCVs, this has been interpreted as evidence for lack of lysosomal fusion with the SCV (Garcia-del Portillo and Finlay, 1995). An alternative explanation for the exclusion of CI M6PR from the SCV is that CI-MPR is recruited to the nascent SCV but is then efficiently removed in a SNX1-dependent manner. Together with studies showing delivery of lysosomal content markers to the SCV this supports a model in which SCVs can fuse with lysosomes during biogenesis (Oh et al., 1996; Drecktrah et al., 2007).

Unlike SNX1, SNX3 does not contain a BAR domain or interact with retromer. However, it also localizes to the endocytic pathway and has been shown to function in sorting and membrane invagination within multivesicular bodies (Pons et al., 2008; Xu and Hensel, 2010). In Salmonella-infected cells, SNX3 transiently localizes to SCVs at early times post invasion $(10 \mathrm{~min})$ and thereafter to membrane tubules formed 30-60 min post invasion (Braun et al., 2010). In SNX3 depleted cells, delivery of Rab7 and Lamp1 to the SCV is impeded, indicating a requirement for this SNX in SCV maturation (Braun et al., 2010). Since SopB is required for formation of both SNX1 and SNX3 tubules (Bujny et al., 2008; Braun et al., 2010), modulation of phosphoinositides in the SCV membrane by this T3SS1 effector appears to be a prerequisite for SCV biogenesis.

Another T3SS1 protein implicated in early SCV biogenesis is SptP, a bifunctional protein that functions both as a GTPase activating protein (GAP) and as a tyrosine phosphatase (Fu and Galan, 1999; Kubori and Galan, 2003; Patel and Galan, 2006; Humphreys et al., 2009). The GAP activity down-regulates Cdc42 and Rac1 and is required for termination of membrane ruffling ( $\mathrm{Fu}$ and Galan, 1999; Kubori and Galan, 2003; Patel and Galan, 2006). Subsequently, SptP dephosphorylates valosin-containing protein (VCP), a member of the AAA protein family that functions in a variety of physiological processes (Humphreys et al., 2009). However, while VCP and its adaptors (p47 and Ufd1) are required for efficient SCV maturation their functions during this process have not been elucidated.

\section{INTERMEDIATE VACUOLE DEVELOPMENT - A OUESTION OF POSITION}

In common with many other intracellular pathogens, including Chlamydia and a variety of viruses, Salmonella use dyneinmediated transport along microtubules to reach a juxtanuclear position adjacent to the microtubule organizing center (MTOC). It is hypothesized that this location is important for access to a supply of cellular and/or pathogen components delivered by dynein motors traveling along microtubules (Wileman, 2007). Movement of the SCV to the MTOC, and maintenance at that position, are multi-factorial processes involving at least three T3SS2 effectors (SseF, SseG, and SifA) as well as two T3SS1 effectors (SipA and SopB; Salcedo and Holden, 2003; Abrahams et al., 2006; Deiwick et al., 2006; Brawn et al., 2007; Wasylnka et al., 2008). Salmonella strains deficient in $s s e F$ or $s s e G$ display a predominantly scattered distribution and recruitment of the dynein motor complex to the SCV requires SseF (Abrahams et al., 2006). Although the mechanism of action of these two effectors remains undetermined, they form a structural and functional interaction that is required for maintenance of the juxtanuclear position (Deiwick et al., 2006). Intriguingly, the actin based motor, non-muscle myosin II, is also implicated in SCV positioning (Wasylnka et al., 2008). Again this process is not well understood, however, myosin II activity may be modulated locally on the SCV via a process involving SopB phosphatase activity and activation of the Rho kinase (ROCK; Wasylnka et al., 2008). How the myosin-actin and 
dynein-microtubule driven processes complement or overlap with one another in SCV relocation has not been addressed.

\section{LATE VACUOLE DEVELOPMENT - HOLDING STILL AND EXTENDING}

During the later stages of infection ( $>6 \mathrm{~h}$ post invasion) SCVs are maintained at the MTOC/juxtanuclear region through the actions of effectors that modulate both microtubule and actin based transport (Abrahams et al., 2006; Deiwick et al., 2006). At the same time membrane tubules formed on the cytosolic surface of the SCV extend rapidly along microtubules toward the cell periphery (Drecktrah et al., 2008). To maintain the position of the SCV while simultaneously generating a dynamic tubular network, the bacteria uses a set of effector proteins that exhibit both co-operative and antagonistic activities.

Undoubtedly the best understood T3SS2 effector is SifA, which is required for the formation of Sif tubules (Stein et al., 1996), the most prominent tubular structures induced by Salmonella. Sif formation involves at least two host cell proteins; the plus end directed microtubule motor kinesin-1 and the kinesin-binding protein SKIP (SifA and kinesin-interacting protein; Boucrot et al., 2005; Dumont et al., 2010). Kinesin recruitment to the SCV and its subsequent activation are critical steps in late SCV maturation [for review see (Leone and Meresse, 2011)]. Recruitment of kinesin does not require SifA, but is instead driven by the T3SS2 effector PipB2, although subsequently, SifA modulates the level of kinesin on the SCV via interaction with SKIP (Henry et al., 2006). In the absence of SifA or SKIP, SCV s accumulate excess kinesin and the membrane becomes compromised resulting in release of the bacteria into the cytoplasm (Beuzon et al., 2000; Dumont et al., 2010). In uninfected mammalian cells SKIP binds to the GTPbound active form of Rab9, a small GTPase localized to endosomes, and is involved in positioning of LE/Lys (Dumont et al., 2010). In infected cells, SifA prevents the interaction of SKIP with Rab9 and, since SKIP specifically interacts with active GTP-bound Rab9, may antagonize Rab9 activity (Jackson et al., 2008). Alternatively, by binding SKIP, SifA may increase the amount of GTP-Rab9 that is available to interact with an alternate target. Rab9 normally regulates retrograde transport from late endosomes to the TGN but it has also been implicated in the formation of lysosome related organelles (LRO), such as melanosomes and platelet dense bodies (Kloer et al., 2010). During LRO formation GTP-Rab9 interacts with a protein complex (BLOC-3; Kloer et al., 2010), and perhaps a similar mechanism is involved in the formation of Sifs, which could also be considered a form of LRO.

Another effector that co-operates with SifA in late SCV biogenesis is the T3SS1 effector SipA. In cells infected with Salmonella lacking SipA, SifA is mislocalized and SCV s are redistributed to the cell periphery (Brawn et al., 2007). The SCV positioning activity of SipA is located in the N-terminal domain of the protein and when expressed exogenously in epithelial cells this domain can associate with LE/Lys and promote clustering in the juxtanuclear region (Brawn et al., 2007). In addition, the actin binding activity of SipA, which is located in the C-terminal domain, also has a role in late SCV biogenesis, being required for accumulation of Factin around the SCV (Meresse et al., 2001; Guignot et al., 2004). This SipA-dependent actin accumulation is likely a result of its ability to inhibit actin disassembly (McGhie et al., 2004). Indeed, depolymerization of the actin cytoskeleton results in redistribution of SCVs toward the cell periphery, although the exact role of actin accumulation around the SCV has not been well studied (Wasylnka et al., 2008).

Another T3SS2 effector protein involved in SCV/Sif biogenesis and stability is SseJ, a protein with some homology to glycerophospholipid:cholesterol acyltransferases (GCATs; Lossi et al., 2008). These enzymes are involved in cholesterol esterification and lipid body formation. SseJ can esterify cholesterol in vitro, in cells and during infection and SCVs and Sifs are enriched in cholesterol (Nawabi et al., 2008). Activation of SseJ depends upon binding GTP-bound RhoA and, although many bacterial effectors are known to manipulate GTPases, this is the first instance of an effector being regulated by the activity of a host GTPase (Christen et al., 2009). Intriguingly, SifA can interact specifically with GDP-bound RhoA, acting as a GEF, and it is possible that SifA, SKIP, SseJ, and RhoA co-operatively promote host membrane tubulation (Ohlson et al., 2008; Vinh et al., 2010). In S. enterica serovar Typhi, the causative agent of human typhoid fever, sseJ is a pseudogene. Surprisingly, when Typhi is trans-complemented with the functional Typhimurium sseJ gene there is a significant decrease in the level of toxicity of the organism to cultured epithelial cells (Trombert et al., 2010). This finding suggests that perhaps loss of SseJ activity is one factor that has contributed to the development of Typhi as an organism associated with systemic disease.

Live-cell imaging studies at later time points ( $>6 \mathrm{~h}$ post invasion) have revealed that formation of Salmonella-induced tubules may involve interactions with the secretory as well as endolysosomal pathways. Thus, although the majority of tubules are highly enriched in Lamp1, a subpopulation is characterized by the presence of the secretory carrier membrane proteins SCAMP2 and SCAMP3 (Mota et al., 2009). These large tetraspan proteins are found predominantly on post-Golgi endocytic and exocytic membranes, but can also be found on PI(3)P enriched endosomes and perhaps function in recycling of endocytosed receptors (Singleton et al., 1997; Castle and Castle, 2005; Aoh et al., 2009). Salmonellainduced SCAMP3 tubules (SISTs) are observed in close association with the Lamp1 positive Sifs and similarly contain multiple T3SS2 effectors (PipB, PipB2, SifA, SseG, SseJ; Mota et al., 2009). Also described recently are LAMP1 negative tubules (LNTs) that may be precursors to Sifs and SISTs and contribute to membrane stability of the SCV (Schroeder et al., 2010). LNTs have been shown in only low numbers of epithelial cells infected with WT Salmonella and it is possible that the T3SS2 effector SopD limits their production under these conditions (Schroeder et al., 2010). The function of all of the Salmonella-induced tubules remains unknown, and indeed it is unclear whether any of them are even formed in vivo. One possibility is that Salmonella might use them to intercept host cell trafficking for nutritional or membrane requirements (Mota et al., 2009).

\section{ESCAPE FROM THE VACUOLE AND THE HOST CELL?}

Although the SCV is considered the primary intracellular niche for Salmonella the bacteria can also be found in the cytoplasm. 
In some cells, such as macrophages, the cytosol is a lethal environment for Salmonella, however, in epithelial cells the cytoplasm supports robust growth (Beuzon et al., 2002; Brumell et al., 2002; Knodler et al., 2010). Intriguingly, this cytosolic stage may serve as a critical transition step that precedes egress into the extracellular environment (Knodler et al., 2010), although this vital stage of the host-pathogen relationship is virtually unstudied for Salmonella. We have recently addressed this question using a polarized epithelial cell model and found that Salmonella take advantage of a host mechanism known as extrusion, which is normally used to remove dead or dying cells during the turnover of the intestinal epithelium (Knodler et al., 2010). The first step in this process seems to be escape from the SCV into the cytoplasm, where bacterial replication far exceeds that occurring within SCVs. The cytoplasmic bacteria also express different virulence genes compared to vacuolar bacteria. Specifically, instead of expressing genes encoding for T3SS2 these bacteria are induced for T3SS1 and flagellar motility. The significance of this is apparent when the infected cell is extruded from the monolayer, and in the process releases a large number of invasive (T3SS1 induced and motile) bacteria. In vivo these extruded bacteria may either infect adjacent cells, in order to repeat the intracellular cycle, or be shed into the environment. Thus, although the SCV is an important intracellular niche for Salmonella, there may be times when escape from the SCV and growth in the cytosol is just as important for the infectious cycle.

\section{AUTOPHAGY}

Autophagy (to eat oneself) is a tightly regulated process that is crucial for normal cell homeostasis and is the major mechanism by which cells reallocate nutrients from non-essential processes to essential processes during starvation. In essence it involves degradation of intracellular components via the lysosome. Eukaryotic cells also use a modified form of autophagy, known as xenophagy (to eat foreign matter), to control and/or degrade intracellular bacteria and viruses. In the case of Salmonella autophagy appears to have an important role in controlling escape from the SCV. In infected HeLa cells microtubule-associated protein light chain 3 (LC3), a protein often used to monitor autophagy, as well as the ubiquitin binding adaptor proteins p62 and NDP52 are present on approximately $20 \%$ of SCVs $1 \mathrm{~h}$ p.i. (Birmingham and Brumell, 2006; Birmingham et al., 2006; Cemma et al., 2011). Subsequently, bacteria are associated with ubiquitinated proteins (Birmingham and Brumell, 2006; Birmingham et al., 2006). The recruitment of LC3 to the SCV seems to involve similar mechanisms as involved in macroautophagy, and is dependent on lipidation of LC3. The lipidation step requires Atg7, Atg3, and Atg5 enzymes and in cells

\section{REFERENCES}

Abrahams, G. L., Muller, P., and Hensel, M. (2006). Functional dissection of SseF, a type III effector protein involved in positioning the salmonella-containing vacuole. Traffic 7, 950-965.

Aiastui, A., Pucciarelli, M. G., and Garcia-Del Portillo, F. (2010). Salmonella enterica serovar typhimurium invades fibroblasts by multiple routes differing from the entry into epithelial cells. Infect. Immun. 78, 2700-2713.

Aoh, Q. L., Castle, A. M., Hubbard, C. H., Katsumata, O., and Castle, J. D. (2009). SCAMP3 negatively regulates epidermal growth factor receptor degradation and promotes receptor recycling. Mol. Biol. Cell 20, 1816-1832.

Bakowski, M. A., Braun, V., Lam, G. Y., Yeung, T., Heo, W. D., Meyer, T., Finlay, B. B., Grinstein, lacking these LC3 recruitment is defective and there is increased cytosolic replication of Salmonella (Birmingham et al., 2006; Kageyama et al., 2011). LC3 recruitment is preceeded by the association of an Atg9L1-positive autophagosome-like double membrane structure around the still-intact SCV as early as $30 \mathrm{~min}$ after infection (Kageyama et al., 2011). It has been proposed that this isolation membrane, which ultimately becomes the xenophagosome, is formed through an Atg9L1-dependent mechanism, and potentially requires PI3-kinase and the ULK1 complex (Kageyama et al., 2011). The LC3 lipidation machinery is apparently recruited independently of these proteins via an unknown targeting mechanism. According to this model LC3 is then involved in closing the isolation membrane thus forming the xenophagosome (Kageyama et al., 2011). How the autophagy pathway recognizes the SCV is not completely understood although it is dependent on the T3SS1, and it appears that membrane damage caused by the secretion system is an important trigger (Birmingham and Brumell, 2006).

\section{CONCLUSION}

The co-evolution of Salmonella serovars and their vertebrate hosts has resulted in the development of a tightly interwoven relationship as illustrated by the complexity of host-pathogen interactions seen at a single cell level. The mechanisms by which Salmonella invade non-phagocytic vertebrate cells and subsequently establish a replicative environment have been extensively investigated. Many of the molecular interactions between host and pathogen have been elucidated in recent years. Nevertheless, many important questions remain unanswered. How do Salmonella avoid host cell defenses such as phagolysosomal fusion? How do Salmonella complete the intracellular cycle and ensure release and/or transmission? What determines whether the bacteria escape into the cytosol or get targeted for xenophagy? Most of the emphasis in the last 10-15 years has been on T3SS1-mediated invasion and subsequent SCV biogenesis, however, it is now clear that Salmonella can use a variety of mechanisms to invade non-phagocytic cells and that the SCV is not the only intracellular niche. In order to address these questions, and advance our understanding of this fascinating pathogen, we may have to turn to alternate model systems, such as polarized epithelial cultures, and consider T3SS1-independent mechanisms of invasion as well as the canonical T3SS1-mediated invasion.

\section{ACKNOWLEDGMENTS}

Work in the author's laboratory is funded by the Intramural Research Program (DIR) of the National Institute of Allergy and Infectious Diseases, National Institutes of Health (NIAID/NIH).
S., and Brumell, J. H. (2010). The phosphoinositide phosphatase SopB manipulates membrane surface charge and trafficking of the Salmonella-containing vacuole. Cell Host Microbe 7, 453-462.

Beuzon, C. R., Meresse, S., Unsworth, K. E., Ruiz-Albert, J., Garvis, S., Waterman, S. R., Ryder, T. A., Boucrot, E., and Holden, D. W. (2000). Salmonella maintains the integrity of its intracellular vacuole through the action of SifA. EMBO J. 19 , 3235-3249.

Beuzon, C. R., Salcedo, S. P., and Holden, D. W. (2002). Growth and killing of a Salmonella enterica serovar Typhimurium sifA mutant strain in the cytosol of different host cell lines. Microbiology 148, 2705-2715.

Birmingham, C. L., and Brumell, J. H. (2006). Autophagy recognizes intracellular Salmonella enterica serovar 
Typhimurium in damaged vacuoles. Autophagy 2, 156-158.

Birmingham, C. L., Smith, A. C., Bakowski, M. A., Yoshimori, T., and Brumell, J. H. (2006). Autophagy controls Salmonella infection in response to damage to the Salmonella-containing vacuole. $J$. Biol. Chem. 281, 11374-11383.

Boucrot, E., Henry, T., Borg, J. P., Gorvel, J. P., and Meresse, S. (2005). The intracellular fate of Salmonella depends on the recruitment of kinesin. Science 308, 1174-1178.

Braun, V., Wong, A., Landekic, M., Hong, W. J., Grinstein, S., and Brumell, J. H. (2010). Sorting nexin 3 (SNX3) is a component of a tubular endosomal network induced by Salmonella and involved in maturation of the Salmonellacontaining vacuole. Cell. Microbiol. 12, 1352-1367.

Brawn, L. C., Hayward, R. D., and Koronakis, V. (2007). Salmonella SPI1 effector SipA persists after entry and cooperates with a SPI2 effector to regulate phagosome maturation and intracellular replication. Cell Host Microbe 1, 63-75.

Brown, M. D., Bry, L., Li, Z., and Sacks, D. B. (2007). IQGAP1 regulates Salmonella invasion through interactions with actin, Racl, and Cdc42. J. Biol. Chem. 282, 30265-30272.

Brumell, J. H., Tang, P., Zaharik, M. L., and Finlay, B. B. (2002). Disruption of the Salmonella-containing vacuole leads to increased replication of Salmonella enterica serovar typhimurium in the cytosol of epithelial cells. Infect. Immun. 70, 3264-3270.

Bujny, M. V., Ewels, P. A., Humphrey, S., Attar, N., Jepson, M. A., and Cullen, P. J. (2008). Sorting nexin-1 defines an early phase of Salmonellacontaining vacuole-remodeling during Salmonella infection. J. Cell. Sci. 121, 2027-2036.

Carlton, J., Bujny, M., Peter, B. J., Oorschot, V. M., Rutherford, A., Mellor, H., Klumperman, J., Mcmahon, H. T., and Cullen, P. J. (2004). Sorting nexin-1 mediates tubular endosome-to-TGN transport through coincidence sensing of high- curvature membranes and 3-phosphoinositides. Curr. Biol. 14, 1791-1800.

Castle, A., and Castle, D. (2005). Ubiquitously expressed secretory carrier membrane proteins (SCAMPs) 1-4 mark different pathways and exhibit limited constitutive trafficking to and from the cell surface. J. Cell. Sci. $118,3769-3780$.
Cemma, M., Kim, P. K., and Brumell, J. H. (2011). The ubiquitin-binding adaptor proteins p62/SQSTM1 and NDP52 are recruited independently to bacteria-associated microdomains to target Salmonella to the autophagy pathway. Autophagy 7, 341-345.

Christen, M., Coye, L. H., Hontz, J. S., Larock, D. L., Pfuetzner, R. A., Megha, and Miller, S. I. (2009). Activation of a bacterial virulence protein by the GTPase RhoA. Sci. Signal. 2, ra71.

Cirillo, D. M., Heffernan, E. J., Wu, L., Harwood, J., Fierer, J., and Guiney, D. G. (1996). Identification of a domain in Rck, a product of the Salmonella typhimurium virulence plasmid, required for both serum resistance and cell invasion. Infect. Immun. 64, 2019-2023.

Clayton, D. J., Bowen, A. J., Hulme, S. D., Buckley, A. M., Deacon, V. L., Thomson, N. R., Barrow, P. A., Morgan, E., Jones, M. A., Watson, M., and Stevens, M. P. (2008). Analysis of the role of 13 major fimbrial subunits in colonisation of the chicken intestines by Salmonella enterica serovar Enteritidis reveals a role for a novel locus. BMC Microbiol. 8, 228.

Corrotte, M., Nyguyen, A. P., Harlay, M. L., Vitale, N., Bader, M. F., and Grant, N. J. (2010). Ral isoforms are implicated in Fc gamma R-mediated phagocytosis: activation of phospholipase D by RalA. J. Immunol. 185, 2942-2950.

Criss, A. K., Ahlgren, D. M., Jou, T. S., Mccormick, B. A., and Casanova, J. E. (2001). The GTPase Racl selectively regulates Salmonella invasion at the apical plasma membrane of polarized epithelial cells. J. Cell. Sci. 114, 1331-1341.

Dai, S., Zhang, Y., Weimbs, T., Yaffe, M. B., and Zhou, D. (2007). Bacteriagenerated PtdIns(3)P recruits VAMP8 to facilitate phagocytosis. Traffic 8, 1365-1374.

Deiwick, J., Salcedo, S. P., Boucrot, E., Gilliland, S. M., Henry, T., Petermann, N., Waterman, S. R., Gorvel, J. P., Holden, D. W., and Meresse, S. (2006). The translocated Salmonella effector proteins SseF and SseG interact and are required to establish an intracellular replication niche. Infect. Immun. 74, 6965-6972.

Drecktrah, D., Knodler, L. A., Galbraith, K., and Steele-Mortimer, O. (2005) The Salmonella SPI1 effector SopB stimulates nitric oxide production long after invasion. Cell. Microbiol. 7, 105-113.

Drecktrah, D., Knodler, L. A., Howe, D., and Steele-Mortimer, O. (2007).
Salmonella trafficking is defined by continuous dynamic interactions with the endolysosomal system. Traffic 8, 212-225.

Drecktrah, D., Levine-Wilkinson, S., Dam, T., Winfree, S., Knodler, L. A. Schroer, T. A., and Steele-Mortimer, O. (2008). Dynamic behavior of Salmonella-induced membrane tubules in epithelial cells. Traffic 9, 2117-2129.

Dukes, J. D., Lee, H., Hagen, R., Reaves, B. J., Layton, A. N., Galyov, E. E., and Whitley, P. (2006). The secreted Salmonella dublin phosphoinositide phosphatase, SopB, localizes to PtdIns(3)P-containing endosomes and perturbs normal endosome to lysosome trafficking. Biochem. J. 395, 239-247.

Dumont, A., Boucrot, E., Drevensek, S., Daire, V., Gorvel, J. P., Pous, C., Holden, D. W., and Meresse, S. (2010). SKIP, the host target of the Salmonella virulence factor SifA, promotes kinesin-1-dependent vacuolar membrane exchanges. Traffic 11, 899-911.

Dunn, J. D., and Valdivia, R. H. (2010). Uncivil engineers: Chlamydia, Salmonella and Shigella alter cytoskeleton architecture to invade epithelial cells. Future Microbiol. 5, 1219-1232.

Edwards, R. A., Olsen, G. J., and Maloy, S. R. (2002). Comparative genomics of closely related salmonellae. Trends Microbiol. 10, 94-99.

Fu, Y., and Galan, J. E. (1999). A salmonella protein antagonizes Rac- 1 and Cdc42 to mediate host-cell recovery after bacterial invasion. Nature 401, 293-297.

Garcia-del Portillo, F., and Finlay, B. B. (1995). Targeting of Salmonella typhimurium to vesicles containing lysosomal membrane glycoproteins bypasses compartments with mannose 6-phosphate receptors. J. Cell Biol. 129, 81-97.

Garcia-del Portillo, F., Zwick, M. B. Leung, K. Y., and Finlay, B. B. (1993). Salmonella induces the formation of filamentous structures containing lysosomal membrane glycoproteins in epithelial cells. Proc. Natl. Acad. Sci. U.S.A. 90, 10544-10548.

Gauthier-Rouviere, C., Vignal, E., Meriane, M., Roux, P., Montcourier, P., and Fort, P. (1998). RhoG GTPase controls a pathway that independently activates Rac1 and Cdc42Hs. Mol. Biol. Cell 9, 1379-1394.

Geddes, K., Cruz, F., and Heffron, F. (2007). Analysis of cells targeted by Salmonella type III secretion in vivo. PLoS Pathog. 3, e196. doi: 10.1371/journal.ppat.0030196
Gerlach, R. G., Claudio, N., Rohde, M., Jackel, D., Wagner, C., and Hensel, M. (2008). Cooperation of Salmonella pathogenicity islands 1 and 4 is required to breach epithelial barriers. Cell. Microbiol. 10, 2364-2376.

Gordon, M. A. (2008). Salmonella infections in immunocompromised adults. J. Infect. 56, 413-422.

Guignot, J., Caron, E., Beuzon, C., Bucci, C., Kagan, J., Roy, C., and Holden, D. W. (2004). Microtubule motors control membrane dynamics of Salmonella-containing vacuoles. J. Cell. Sci. 117, 1033-1045.

Hardt, W. D., Chen, L. M., Schuebel, K. E., Bustelo, X. R., and Galan, J. E. (1998). S. typhimurium encodes an activator of Rho GTPases that induces membrane ruffling and nuclear responses in host cells. Cell 93, 815-826.

Hayward, R. D., and Koronakis, V. (1999). Direct nucleation and bundling of actin by the SipC protein of invasive Salmonella. EMBO J. 18, 4926-4934.

Hayward, R. D., and Koronakis, V. (2002). Direct modulation of the host cell cytoskeleton by Salmonella actin-binding proteins. Trends Cell Biol. 12, 15-20.

Heffernan, E. J., Harwood, J., Fierer, J., and Guiney, D. (1992a). The Salmonella typhimurium virulence plasmid complement resistance gene rck is homologous to a family of virulence-related outer membrane protein genes, including pagC and ail. J. Bacteriol. 174, 84-91.

Heffernan, E. J., Reed, S., Hackett, J., Fierer, J., Roudier, C., and Guiney, D. (1992b). Mechanism of resistance to complement-mediated killing of bacteria encoded by the Salmonella typhimurium virulence plasmid gene rck. J. Clin. Invest. 90, 953-964.

Henry, T., Couillault, C., Rockenfeller, P., Boucrot, E., Dumont, A., Schroeder, N., Hermant, A., Knodler, L. A., Lecine, P., Steele-Mortimer, O., Borg, J. P., Gorvel, J. P., and Meresse, S. (2006). The Salmonella effector protein PipB2 is a linker for kinesin1. Proc. Natl. Acad. Sci. U.S.A. 103, 13497-13502.

Hernandez, L. D., Hueffer, K., Wenk, M. R., and Galan, J. E. (2004). Salmonella modulates vesicular traffic by altering phosphoinositide metabolism. Science 304, 1805-1807.

Hilpela, P., Vartiainen, M. K., and Lappalainen, P. (2004). Regulation of the actin cytoskeleton by $\mathrm{PI}(4,5) \mathrm{P} 2$ and $\mathrm{PI}(3,4,5) \mathrm{P} 3$. Curr. Top. Microbiol. Immunol. 282, 117-163. 
Hoppe, A. D., and Swanson, J. A. (2004). Cdc42, Rac1, and Rac2 display distinct patterns of activation during phagocytosis. Mol. Biol. Cell 15, 3509-3519.

Huett, A., Leong, J. M., Podolsky, D. K., and Xavier, R. J. (2009). The cytoskeletal scaffold Shank3 is recruited to pathogen-induced actin rearrangements. Exp. Cell Res. 315, 2001-2011.

Humphreys, D., Hume, P. J., and Koronakis, V. (2009). The Salmonella effector SptP dephosphorylates host AAA+ ATPase VCP to promote development of its intracellular replicative niche. Cell Host Microbe 5, 225-233.

Jackson, L. K., Nawabi, P., Hentea, C., Roark, E. A., and Haldar, K. (2008). The Salmonella virulence protein SifA is a G protein antagonist. Proc. Natl. Acad. Sci. U.S.A. 105, 14141-14146.

Jones, B. D., Ghori, N., and Falkow, S. (1994). Salmonella typhimurium initiates murine infection by penetrating and destroying the specialized epithelial $M$ cells of the Peyer's patches. J. Exp. Med. 180, 15-23.

Kageyama, S., Omori, H., Saitoh, T., Sone, T., Guan, J. L., Akira, S., Imamoto, F., Noda, T., and Yoshimori, T. (2011). The LC3 recruitment mechanism is separate from Atg9L1-dependent membrane formation in the autophagic response against Salmonella. Mol. Biol. Cell. doi:10.1091/mbc.E10-110893. [Epub ahead of print].

Katoh, H., and Negishi, M. (2003). RhoG activates Racl by direct interaction with the Dock180binding protein Elmo. Nature 424, 461-464.

Kloer, D. P., Rojas, R., Ivan, V., Moriyama, K., Van Vlijmen, T., Murthy, N., Ghirlando, R., Van Der Sluijs, P., Hurley, J. H., and Bonifacino, J. S. (2010). Assembly of the biogenesis of lysosome-related organelles complex-3 (BLOC-3) and its interaction with Rab9. J. Biol. Chem. 285, 7794-7804.

Knodler, L. A., Finlay, B. B., and SteeleMortimer, O. (2005). The Salmonella effector protein SopB protects epithelial cells from apoptosis by sustained activation of Akt. J. Biol. Chem. 280, 9058-9064.

Knodler, L. A., Vallance, B. A., Celli, J., Winfree, S., Hansen, B., Montero, M., and Steele-Mortimer, O. (2010). Dissemination of invasive Salmonella via bacterial-induced extrusion of mucosal epithelia. Proc. Natl. Acad. Sci. U.S.A. 107, 17733-17738.
Kubori, T., and Galan, J. E. (2003). Temporal regulation of salmonella virulence effector function by proteasome-dependent protein degradation. Cell 115, 333-342.

Lambert, M. A., and Smith, S. G. (2008). The PagN protein of Salmonella enterica serovar Typhimurium is an adhesin and invasin. BMC Microbiol. 8,142 .

Lambert, M. A., and Smith, S. G. (2009). The PagN protein mediates invasion via interaction with proteoglycan. FEMS Microbiol. Lett. 297, 209-216.

Lawley, T. D., Chan, K., Thompson, L. J., Kim, C. C., Govoni, G. R., and Monack, D. M. (2006). Genome-wide screen for Salmonella genes required for long-term systemic infection of the mouse. PLoS Pathog. 2, ell. doi: 10.1371/journal.ppat.0020011

Leone, P., and Meresse, S. (2011). Kinesin regulation by Salmonella. Virulence 2, 63-66.

Lossi, N. S., Rolhion, N., Magee, A. I., Boyle, C., and Holden, D. W. (2008). The Salmonella SPI2 effector SseJ exhibits eukaryotic activator-dependent phospholipase A and glycerophospholipid: cholesterol acyltransferase activity. Microbiology 154, 2680-2688.

Ly, K. T., and Casanova, J. E. (2009). Abelson tyrosine kinase facilitates Salmonella enterica serovar Typhimurium entry into epithelial cells. Infect. Immun. 77, 60-69.

Magalhaes, M. A., and Glogauer, M. (2010). Pivotal Advance: Phospholipids determine net membrane surface charge resulting in differential localization of active Rac1 and Rac2. J. Leukoc. Biol. 87, 545-555.

Majowicz, S. E., Musto, J., Scallan, E., Angulo, F. J., Kirk, M., O’brien, S. J., Jones, T. F., Fazil, A., and Hoekstra, R. M. (2010). The global burden of nontyphoidal Salmonella gastroenteritis. Clin. Infect. Dis. 50, 882-889.

Mallo, G. V., Espina, M., Smith, A. C., Terebiznik, M. R., Aleman, A., Finlay, B. B., Rameh, L. E., Grinstein, S., and Brumell, J. H. (2008). SopB promotes phosphatidylinositol 3-phosphate formation on Salmonella vacuoles by recruiting Rab5 and Vps34. J. Cell Biol. 182, 741-752.

Marlovits, T. C., and Stebbins, C. E. (2010). Type III secretion systems shape up as they ship out. Curr. Opin. Microbiol. 13, 47-52.

McGhie, E. J., Brawn, L. C., Hume, P. J., Humphreys, D., and Koronakis, V. (2009). Salmonella takes control: effector-driven manipulation of the host. Curr. Opin. Microbiol. 12, 117-124.
McGhie, E. J., Hayward, R. D., and Koronakis, V. (2001). Cooperation between actin-binding proteins of invasive Salmonella: SipA potentiates SipC nucleation and bundling of actin. EMBO J. 20, 2131-2139.

McGhie, E. J., Hayward, R. D., and Koronakis, V. (2004). Control of actin turnover by a salmonella invasion protein. Mol. Cell 13, 497-510.

Meresse, S., Unsworth, K. E., Habermann, A., Griffiths, G., Fang, F., Martinez-Lorenzo, M. J., Waterman, S. R., Gorvel, J. P., and Holden, D. W. (2001). Remodelling of the actin cytoskeleton is essential for replication of intravacuolar Salmonella. Cell. Microbiol. 3, 567-577.

Meyerholz, D. K., Stabel, T. J., Ackermann, M. R., Carlson, S. A., Jones, B. D., and Pohlenz, J. (2002). Early epithelial invasion by Salmonella enterica serovar Typhimurium DT104 in the swine ileum. Vet. Pathol. 39, 712-720.

Misselwitz, B., Kreibich, S. K., Rout, S., Stecher, B., Periaswamy, B., and Hardt, W. D. (2010). Salmonella enterica serovar Typhimurium binds to HeLa cells via Fim-mediated reversible adhesion and irreversible type three secretion system 1mediated docking. Infect. Immun. 79, 330-341.

Mota, L. J., Ramsden, A. E., Liu, M., Castle, J. D., and Holden, D. W. (2009). SCAMP3 is a component of the Salmonella-induced tubular network and reveals an interaction between bacterial effectors and postGolgi trafficking. Cell. Microbiol. 11, 1236-1253.

Murli, S., Watson, R. O., and Galan, J. E. (2001). Role of tyrosine kinases and the tyrosine phosphatase $\mathrm{SptP}$ in the interaction of Salmonella with host cells. Cell. Microbiol. 3, 795-810.

Myeni, S. K., and Zhou, D. (2010). The $\mathrm{C}$ terminus of SipC binds and bundles F-actin to promote Salmonella invasion. J. Biol. Chem. 285, 13357-13363.

Nawabi, P., Catron, D. M., and Haldar, K. (2008). Esterification of cholesterol by a type III secretion effector during intracellular Salmonella infection. Mol. Microbiol. 68, 173-185.

Nichols, C. D., and Casanova, J. E. (2010). Salmonella-directed recruitment of new membrane to invasion foci via the host exocyst complex. Curr. Biol. 20, 1316-1320.

Norris, F. A., Wilson, M. P., Wallis, T. S., Galyov, E. E., and Majerus, P. W. (1998). SopB, a protein required for virulence of Salmonella dublin, is an inositol phosphate phosphatase. Proc. Natl. Acad. Sci. U.S.A. 95, 14057-14059.

Oh, Y. K., Alpuche-Aranda, C., Berthiaume, E., Jinks, T., Miller, S. I., and Swanson, J. A. (1996). Rapid and complete fusion of macrophage lysosomes with phagosomes containing Salmonella typhimurium. Infect. Immun. 64, 3877-3883.

Ohlson, M. B., Huang, Z., Alto, N. M., Blanc, M. P., Dixon, J. E., Chai, J., and Miller, S. I. (2008). Structure and function of Salmonella SifA indicate that its interactions with SKIP, SseJ, and RhoA family GTPases induce endosomal tubulation. Cell Host Microbe 4, 434-446.

Patel, J. C., and Galan, J. E. (2006). Differential activation and function of Rho GTPases during Salmonellahost cell interactions. J. Cell Biol. 175, 453-463.

Patel, J. C., Hueffer, K., Lam, T. T., and Galan, J. E. (2009). Diversification of a Salmonella virulence protein function by ubiquitindependent differential localization. Cell 137, 283-294.

Petch, L. A., Bockholt, S. M., Bouton, A., Parsons, J. T., and Burridge, K. (1995). Adhesion-induced tyrosine phosphorylation of the p130 src substrate. J. Cell. Sci. 108 ( Pt 4), 1371-1379.

Pons, V., Luyet, P. P., Morel, E., Abrami, L., Van Der Goot, F. G., Parton, R. G., and Gruenberg, J. (2008). Hrs and SNX3 functions in sorting and membrane invagination within multivesicular bodies. PLoS Biol. 6, e214. doi: 10.1371/journal.pbio.0060214

Pryor, P. R., Mullock, B. M., Bright, N. A., Lindsay, M. R., Gray, S. R., Richardson, S. C., Stewart, A., James, D. E., Piper, R. C., and Luzio, J. P. (2004). Combinatorial SNARE complexes with VAMP7 or VAMP8 define different late endocytic fusion events. EMBO Rep. 5, 590-595.

Radtke, A. L., Wilson, J. W., Sarker, S., and Nickerson, C. A. (2010). Analysis of interactions of Salmonella type three secretion mutants with 3-D intestinal epithelial cells. PLoS One 5, e15750. doi: 10.1371/journal.pone. 0015750

Raffatellu, M., Wilson, R. P., Chessa, D., Andrews-Polymenis, H., Tran, Q T., Lawhon, S., Khare, S., Adams, L. G., and Baumler, A. J. (2005). SipA, SopA, SopB, SopD, and SopE2 contribute to Salmonella enterica serotype typhimurium invasion of epithelial cells. Infect. Immun. 73, 146-154. 
Ramsden, A. E., Holden, D. W., and Mota, L. J. (2007). Membrane dynamics and spatial distribution of Salmonella-containing vacuoles. Trends Microbiol. 15, 516-524.

Rescigno, M., Urbano, M., Valzasina, B., Francolini, M., Rotta, G., Bonasio, R., Granucci, F., Kraehenbuhl, J. P., and Ricciardi-Castagnoli, P. (2001). Dendritic cells express tight junction proteins and penetrate gut epithelial monolayers to sample bacteria. Nat. Immunol. 2, 361-367.

Richter-Dahlfors, A., Buchan, A. M., and Finlay, B. B. (1997). Murine salmonellosis studied by confocal microscopy: Salmonella typhimurium resides intracellularly inside macrophages and exerts a cytotoxic effect on phagocytes in vivo. J. Exp. Med. 186, 569-580.

Rittmeyer, E. N., Daniel, S., Hsu, S. C., and Osman, M. A. (2008). A dual role for IQGAP1 in regulating exocytosis. J. Cell. Sci. 121, 391-403.

Rosselin, M., Abed, N., VirlogeuxPayant, I., Bottreau, E., Sizaret, P. Y., Velge, P., and Wiedemann, A. (2010). Heterogeneity of T3SS1-independent entry mechanisms used by Salmonella Enteritidis to invade different cell types. Microbiology 157, 839-847.

Salcedo, S. P., and Holden, D. W. (2003). SseG, a virulence protein that targets Salmonella to the Golgi network. EMBO J. 22, 5003-5014.

Salcedo, S. P., Noursadeghi, M., Cohen, J., and Holden, D. W. (2001). Intracellular replication of Salmonella typhimurium strains in specific subsets of splenic macrophages in vivo. Cell. Microbiol. 3, 587-597.

Samudrala, R., Heffron, F., and Mcdermott, J. E. (2009). Accurate prediction of secreted substrates and identification of a conserved putative secretion signal for type III secretion systems. PLoS Pathog. 5, e1000375. doi: 10.1371/journal.ppat.1000375

Schroeder, N., Henry, T., De Chastellier, C., Zhao, W., Guilhon, A. A., Gorvel, J. P., and Meresse, S. (2010). The virulence protein SopD2 regulates membrane dynamics of Salmonella-containing vacuoles. PLoS Pathog. 6, e1001002. doi: 10.1371/journal.ppat.1001002

Schuetz, G., Rosario, M., Grimm, J., Boeckers, T. M., Gundelfinger, E. D., and Birchmeier, W. (2004). The neuronal scaffold protein Shank3 mediates signaling and biological function of the receptor tyrosine kinase Ret in epithelial cells. J. Cell Biol. 167, 945-952.

Scott, C. C., Dobson, W., Botelho, R. J., Coady-Osberg, N., Chavrier, P., Knecht, D. A., Heath, C., Stahl, P., and Grinstein, S. (2005). Phosphatidylinositol-4,5bisphosphate hydrolysis directs actin remodeling during phagocytosis. J. Cell Biol. 169, 139-149.

Sharma, A., and Mayer, B. J. (2008). Phosphorylation of p130Cas initiates Rac activation and membrane ruffling. BMC Cell Biol. 9, 50. doi: 10.1186/1471-2121-9-50

Shi, J., and Casanova, J. E. (2006). Invasion of host cells by Salmonella typhimurium requires focal adhesion kinase and p130Cas. Mol. Biol. Cell 17, 4698-4708.

Singleton, D. R., Wu, T. T., and Castle, J. D. (1997). Three mammalian SCAMPs (secretory carrier membrane proteins) are highly related products of distinct genes having similar subcellular distributions. $J$. Cell. Sci. 110, 2099-2107.

Steele-Mortimer, O., Knodler, L. A., Marcus, S. L., Scheid, M. P., Goh, B., Pfeifer, C. G., Duronio, V., and Finlay, B. B. (2000). Activation of Akt/protein kinase B in epithelial cells by the Salmonella typhimurium effector sigD. J. Biol. Chem. 275, 37718-37724.

Steele-Mortimer, O., Meresse, S., Gorvel, J. P., Toh, B. H., and Finlay, B. B. (1999). Biogenesis of Salmonella typhimurium-containing vacuoles in epithelial cells involves interactions with the early endocytic pathway. Cell. Microbiol. 1, 33-49.

Stein, M. A., Leung, K. Y., Zwick, M., Garcia-Del Portillo, F., and Finlay, B. B. (1996). Identification of a Salmonella virulence gene required for formation of filamentous structures containing lysosomal membrane glycoproteins within epithelial cells. Mol. Microbiol. 20, 151-164.

Stender, S., Friebel, A., Linder, S., Rohde, M., Mirold, S., and Hardt, W. D. (2000). Identification of SopE2 from Salmonella typhimurium, a conserved guanine nucleotide exchange factor for Cdc42 of the host cell. Mol. Microbiol. 36, 1206-1221.

Stevens, M. P., Friebel, A., Taylor, L. A., Wood, M. W., Brown, P. J., Hardt, W. D., and Galyov, E. E. (2003). A Burkholderia pseudomallei type III secreted protein, BopE, facilitates bacterial invasion of epithelial cells and exhibits guanine nucleotide exchange factor activity. J. Bacteriol. 185, 4992-4996.

Terebiznik, M. R., Vieira, O. V., Marcus, S. L., Slade, A., Yip, C. M., Trimble, W. S., Meyer, T., Finlay, B. B., and Grinstein, S. (2002). Elimination of host cell PtdIns(4,5) $\mathrm{P}(2)$ by bacterial SigD promotes membrane fission during invasion by Salmonella. Nat. Cell Biol. 4, 766-773.

Trombert, A. N., Berrocal, L., Fuentes, J. A., and Mora, G. C. (2010). S. Typhimurium sseJ gene decreases the S. Typhi cytotoxicity toward cultured epithelial cells. BMC Microbiol. 10, 312. doi: 10.1186/14712180-10-312

Unsworth, K. E., Way, M., Mcniven, M., Machesky, L., and Holden, D. W. (2004). Analysis of the mechanisms of Salmonella-induced actin assembly during invasion of host cells and intracellular replication. Cell. Microbiol. 6, 1041-1055.

Van Weering, J. R., Verkade, P., and Cullen, P. J. (2010). SNXBAR proteins in phosphoinositidemediated, tubular-based endosomal sorting. Semin. Cell Dev. Biol. 21, 371-380.

Vinh, D. B., Ko, D. C., Rachubinski, R. A., Aitchison, J. D., and Miller, S. I. (2010). Expression of the Salmonella spp. virulence factor SifA in yeast alters Rhol activity on peroxisomes. Mol. Biol. Cell 21, 3567-3577.

Wallis, T. S., Starkey, W. G., Stephen, J., Haddon, S. J., Osborne, M. P., and Candy, D. C. (1986). The nature and role of mucosal damage in relation to Salmonella typhimuriuminduced fluid secretion in the rabbit ileum. J. Med. Microbiol. 22, 39-49.

Wasylnka, J. A., Bakowski, M. A., Szeto, J., Ohlson, M. B., Trimble, W. S., Miller, S. I., and Brumell, J. H. (2008). Role for myosin II in regulating positioning of Salmonella-containing vacuoles and intracellular replication. Infect. Immun. 76, 2722-2735.

Waterman, S. R., and Holden, D. W. (2003). Functions and effectors of the Salmonella pathogenicity island 2 type III secretion system. Cell. Microbiol. 5, 501-511.

Wileman, T. (2007). Aggresomes and pericentriolar sites of virus assembly: cellular defense or viral design? Annu. Rev. Microbiol. 61, 149-167.

Worby, C. A., and Dixon, J. E. (2002). Sorting out the cellular functions of sorting nexins. Nat. Rev. Mol. Cell Biol. 3, 919-931.

$\mathrm{Xu}, \mathrm{X}$., and Hensel, M. (2010). Systematic analysis of the SsrAB virulon of Salmonella enterica. Infect. Immun. 78, 49-58.

Zhou, D., and Galan, J. (2001). Salmonella entry into host cells: the work in concert of type III secreted effector proteins. Microbes Infect. 3, 1293-1298.

Zhou, D., Mooseker, M. S., and Galan, J. E. (1999). Role of the S. typhimurium actin-binding protein SipA in bacterial internalization. Science 283, 2092-2095.

Conflict of Interest Statement: The authors declare that the research was conducted in the absence of any commercial or financial relationships that could be construed as a potential conflict of interest.

Received: 07 March 2011; accepted: 19 May 2011; published online: 03 June 2011.

Citation: Malik-Kale P, Jolly CE, Lathrop $S$, Winfree $S$, Luterbach $C$ and SteeleMortimer O (2011) Salmonella-at home in the host cell. Front. Microbio. 2:125. doi: 10.3389/fmicb.2011.00125

This article was submitted to Frontiers in Cellular and Infection Microbiology, a specialty of Frontiers in Microbiology. Copyright (c) 2011 Malik-Kale, Jolly, Lathrop, Winfree, Luterbach and SteeleMortimer. This is an open-access article subject to a non-exclusive license between the authors and Frontiers Media $S A$, which permits use, distribution and reproduction in other forums, provided the original authors and source are credited and other Frontiers conditions are complied with. 